\title{
Application of Distributed Optical Fiber Sensing Technology in Surrounding Rock Deformation Control of TBM-Excavated Coal Mine Roadway
}

\author{
Bin Tang $(\mathbb{D})$ and Hua Cheng $(\mathbb{D}$ \\ School of Civil Engineering and Architecture, Anhui University of Science and Technology, 168 Taifeng St., Huainan 232001, China \\ Correspondence should be addressed to Hua Cheng; hcheng@aust.edu.cn
}

Received 16 August 2017; Accepted 26 December 2017; Published 22 February 2018

Academic Editor: Romeo Bernini

Copyright (C) 2018 Bin Tang and Hua Cheng. This is an open access article distributed under the Creative Commons Attribution License, which permits unrestricted use, distribution, and reproduction in any medium, provided the original work is properly cited.

\begin{abstract}
After roadway excavation, the deformation and failure of roadway surrounding rocks typically results in roadway damage or collapse. Conventional monitoring techniques, such as extensometers, stress meters, and convergence stations, are only capable to detect the stress or strain data with the shallow layers of surrounding rocks, and they require arduous manual works. Moreover, in the abovementioned monitoring techniques, the monitoring instruments are installed behind the excavation face; therefore, the strain and deformation occurring in front of excavation face cannot be detected. In order to eliminate these shortcomings, an innovative monitoring system for surrounding rock deformation control has been developed base on Brillouin optical time domain reflectometry. Compared with conventional monitoring systems, the proposed system provides a reliable, accurate, and real-time monitoring measure for roadway surrounding rock deformation control over wide extension. The optical fiber sensors are installed in boreholes which are situated ahead of the excavation face; therefore, the sensors can be protected well and the surrounding rock deformation behaviors can be studied. The proposed system has been applied within a TBM-excavated roadway in Zhangji coal mine, China. The surrounding rock deformation behaviors have been detected accurately, and the monitoring results provided essential references for surrounding rock deformation control works.
\end{abstract}

\section{Introduction}

In recent 20 years, as the depletion of coal resources in shallow strata, coal mining operations have been moving to deeper strata [1]. Approximately $60 \%$ of the coal mining works are conducted with a mining depth of over $800 \mathrm{~m}$ in China [2]. Mining works in deep strata are facing the challenges of high ground stresses and complex geological conditions [3]. These emerging problems have resulted in large deformation and failures of surrounding rocks and roadway collapse which seriously threaten the safety of miners and limit output capacity of coal productions. Roadway collapse accounts for $80 \%$ of total coal mine accidents and it contributes $43 \%$ fatalities of miners [4]. The conventional monitoring techniques such as extensometers, stress meters, and convergence stations which have been typically applied in shallow roadways cannot meet the requirement of monitoring works in deep strata due to the lower precision and excessive manual operations.

For overcoming the problems of surrounding rock deformation monitoring in deep coal mines, many emerging measurement techniques have been conducted at working faces of coal mining and roadway excavation in underground coal mines. Zhao et al. conducted damage process monitoring of roadway surrounding rock by using microseismic techniques [5]. Zhao et al. proposed a displacement monitoring methodology of overlying rock layers of coal seam based on fiber Bragg grating (FBG) displacement sensors [6]. Kajzar et al. carried out coal pillar deformation and coal roof monitoring by applying 3D laser technology within underground roadways [7]. Yu et al. investigated surrounding rock deformation and roadway convergence by laser range instruments [8]. Martino and Chandler studied surrounding rock deformation and damage zone evolution behaviors by using a borehole 
camera image [9]. Blümling et al. presented long-term processes of surrounding rock damage by microfocus X-ray tomography [10]. Lubosik et al. proposed measurement techniques for axial forces of rockbolts and rock mass displacement by applying instrumented rock bolts which embedded strain gauges and tensometric sensors [11]. Liu et al. suggested transient electromagnetic method (TEM) for detecting surrounding rock damage zone range and deformation [12]. Erich discussed collapse feature of coal mine roadways by employing seismic reflection investigation method [13].

Despite the fact that the monitoring techniques have gained some advances, the abovementioned monitoring methods are still flawed in some aspects. Microseismic techniques and transient electromagnetic and seismic reflection investigation are able to detect fracture evolution in surrounding rocks, while their accuracy on rock displacement monitoring is unsatisfied (reach to meters). Microfocus Xray tomography is only capable of measure damages in rock samples; therefore, it cannot be applied for in situ monitoring. Compared with fully distributed fiber optic sensor systems, FBG systems call for an excessive number of sensors and that leads to high cost. Moreover, most commercially available interrogators can handle only a fairly small number of FBGs, setting a limit on the number of sensing points, as well as on their density along the fiber [14]. Borehole camera image can detect the damage and fracture within the surrounding rocks while the real-time monitoring cannot be achieved and the image analysis relies on manual operations. Instrumented rock bolt can only be used to measure stress and strain in a shallow section of the surrounding rocks due to the limitation on length of rock bolts (typically less than $2.5 \mathrm{~m}$ ). 3D laser technology provides a high-precision instrument for roadway convergence, while the internal deformation and damage of rocks cannot be measured.

Brillouin optical time domain reflectometry (BOTDR) is a fully distributed sensing technology for distributed strain and temperature measurement along all determined areas with only one optical fiber which is stimulated by laser pulses and therefore many discrete sensors can be replaced [15-17]. BOTDR provides fast and reliable measurements, and it also enables early detection of deformations that may affect the safety of mining operations, thereby allowing to schedule necessary works in advance to mitigate the potential risks. In recent years, BOTDR system has been applied in underground coal mines by many researchers. Naruse et al. conducted BOTDR monitoring in El Teniente mine, Chile. The optical fibers are set within the roadway along the roadway alignment; therefore, the roadway convergence can be measured [18]. Cheng et al. measured the deformation of overlying rock layers of coal seam by employing BOTDR-based monitoring method [19]. Zhang and Wang built a fiber mesh structure on the surface of the roadway and conducted BOTDR strain measurement [20, 21].

In previous BOTDR applications, optical fibers were installed about $5 \mathrm{~m}$ behind the excavation faces of roadways to avoid interference with the installation of supporting structures (rockbolts, cable bolts, steel meshes, etc.). Therefore, only time-dependent deformation can be measured and the immediate deformation which occurs soon after excavation cannot be studied. However, $80 \%$ of total roadway damage and collapse accidents happened near the excavation faces [22-24]. Therefore, the monitoring of the whole section of the roadway, includes a deeper layer of surrounding rocks and excavation faces, has been a crucial issue of ensuring safety production in underground coal mines.

This paper focuses on developing a BOTDR-based monitoring system for surrounding rock control of roadways in underground coal mines. The structure of the monitoring system is modified so that a real-time monitoring of both immediate and time-dependent deformation of surrounding rocks can be detected. In situ monitoring of the system in a roadway is proposed, and the monitoring results are analyzed and compared with measurement results acquired from conventional monitoring techniques.

\section{Development of the BOTDR Monitoring System for Surrounding Rock Control in Underground Coal Mines}

2.1. Basic Principles of the BOTDR Monitoring System. The BOTDR-based monitoring system implements Brillouin scattering, which is a basic physical process representing the interaction effect between light and optical medium in propagation medium $[25,26]$. When light is sent through an optical fiber, most of it propagates along the original direction, a small portion of light departure from the original direction and results in a scattering. There are three types of light scattering in optical fiber: Rayleigh scattering caused by the change of fiber refractive index, Raman scattering induced by optical phonon, and Brillouin scattering produced by acoustic phonon [27]. In Brillouin scattering, the scattered light reaches a peak over its spectrum at a frequency shifted from the pulsed light. This amount of frequency shift is known as Brillouin frequency shift, $v_{B}$ [26].

$$
v_{\mathrm{B}}=\frac{2 n V_{\mathrm{a}}}{\lambda}
$$

where $n$ denotes the effective refractive index of the fiber, $V_{\mathrm{a}}$ indicates the acoustic wave velocity of the fiber core, and $\lambda$ is the vacuum wavelength of the incident light.

$$
V_{\mathrm{a}}=\sqrt{\frac{(1-\mu) E}{(1+\mu)(1-2 \mu) \rho}},
$$

where $E, \mu$, and $\rho$ represent Young's modulus, Poisson ratio, and density of optical fiber, respectively.

If longitudinal strain $\varepsilon$ occurs in the optical fiber, the Brillouin frequency shift $v_{\mathrm{B}}$ changes in proportion to that of the strain. The relation can be expressed as

$$
v_{\mathrm{B}}(\varepsilon)=v_{\mathrm{B}}(0)+\frac{d v_{\mathrm{B}}(\varepsilon)}{d \varepsilon} \varepsilon,
$$

where $v_{\mathrm{B}}(\varepsilon)$ is the drift quantity of Brillouin scattering light frequency when the optical fiber strain occurs, $v_{\mathrm{B}}(0)$ indicates the drift quantity of Brillouin scattering light frequency when the optical fiber does not have strain occurs, $d v_{\mathrm{B}}(\varepsilon) / d \varepsilon$ is the scale factor, and $\varepsilon$ is the fiber axial strain. 
Considering the influence of strain and temperature, the Brillouin frequency shift $v_{\mathrm{B}}$ can be expressed as

$$
v_{\mathrm{B}}(\varepsilon, T)=v_{\mathrm{B}}(0)+\frac{d v_{\mathrm{B}}(\varepsilon)}{d \varepsilon} \varepsilon+\frac{d v_{\mathrm{B}}(T)}{d T} T,
$$

where $v_{\mathrm{B}}(\varepsilon, T)$ is the drift quantity of Brillouin scattering light frequency when the optical fiber strain and temperature changing occur, $v_{\mathrm{B}}(0)$ indicates the drift quantity of Brillouin scattering light frequency when the changing values of strain and temperature are zero, $d v_{\mathrm{B}}(\varepsilon) / d \varepsilon$ is the strain factor, $d v_{\mathrm{B}}(T) / d T$ is the temperature factor, $\varepsilon$ is fiber axial strain, and $T$ is the temperature changing.

For typical optical fibers, the scale factor is $493 \mathrm{MHz} / \%$ (strain) and the temperature factor is $1 \mathrm{MHz} /{ }^{\circ} \mathrm{C}$.

The position where the strain occurs can be detected by analyzing the time interval $(t)$ between launching the pulsed light and receiving the scattered light at the end of the optical fiber. The distance between the position where the strain happens and the end of the optical fiber can be expressed as

$$
Z=\frac{c t}{2 n},
$$

where $Z$ is the distance between the position where the strain happens to the end of the optical fiber, $t$ is the time interval between launching the pulsed light and receiving the scattered light at the end of the optical fiber, $c$ is the light velocity in vacuum, and $n$ is the refractive index of the optical fiber. The basic principle of BOTDR is shown in Figure 1.

2.2. System Structure. The BOTDR-based monitoring system consists of the ground unit and underground unit, as shown in Figure 2. The ground unit is connected with the industrial Ethernet by the monitoring host. The monitoring host processes data obtained from underground monitoring devices and the monitoring results can be shown on various terminals (computer, pad, smartphone, etc.). Devices of the ground unit are connected from each other by single-mode cable, mobile network, or Wi-Fi. The ground unit is also connected with the Internet for remote monitoring.

The underground unit of the monitoring system contains the communication system and BOTDR sensing system. The communication system includes communication substations and industrial switch. The communication substations receive monitoring data from different underground working sites, and the industrial switch connects the communication substations and ground unit by using RS485 interface and MHYV cables.

BOTDR sensing system comprises laser light source, pulse modulation unit, optical heterodyne receiver, electrical heterodyne receiver, digital processor, and optical fiber sensors. The digital processor is linked with the nearest communication substation by the MHYV cable. The average distance of the optical fiber sensor to the monitoring host is $6.9 \mathrm{~km}$.

2.3. Optical Fiber Sensor. The fiber sensors are capable of sensing the temperature and strain over long distances $[29,30]$. While the bare optical fibers are usually used in laboratory tests because they are easily fractured under the deformation of measured structures. Therefore, fiber encapsulation is required before monitoring for optical fiber sensor protection as well as insurance of essential coupling effect between sensors and geotechnical structures [31-34]. Many fiber encapsulation methods have been proposed by researchers, such as PVC or glass fiber-reinforced polymer (GFRP) encapsulation, and steel and wire encapsulation. Usually, PVC or GFRP encapsulation are applied in laboratory tests and soil body monitoring due to their low strength and stiffness. In this paper, steel wire-reinforced optical fiber sensors are applied for two reasons: (1) the optical fiber sensors could be damaged in underground structures under huge tectonic stress and ground stress which can reach up to $30 \mathrm{MPa}$; therefore, only steel wire encapsulation is capable to provide a suitable protection for optical fiber sensors. (2) The optical fiber sensors need to be installed in deep boreholes for surrounding rock deformation measurement, and the steel wire-reinforced fibers are easier to insert into boreholes due to higher stiffness of the steel wire.

Figure 3 shows the structure of the steel wire-reinforced optical fiber sensor. The optical fiber is enclosed by a set of steel wires which provides protection for the fiber, and the steel wires and fiber are sealed by a polymer sheath in order to isolate them from groundwater and dust. Therefore, reliable performance of optical fiber sensors can be obtained in harsh work conditions of the underground working site.

2.4. Laboratory Calibrations. Before conducting in situ monitoring, the optical fiber sensors need to be calibrated in the laboratory for understanding their mechanical behaviors. The calibration works include strain calibration and temperature calibration.

Stain calibration was conducted by a fiber tensile machine. An optical fiber in length of $1.2 \mathrm{~m}$ is stretched on the tensile machine; the strain of fiber and corresponding Brillouin frequency shift are recorded for analysis. Therefore, the linear relationship between strain and Brillouin frequency shift can be obtained. The strain factor of the steelreinforced optical fibers which are used in this project is $499.8 \mathrm{MHz} / \%$. The laboratory calibration relationship between strain and Brillouin frequency shift is shown in Figure 4.

Temperature calibration is implemented by a water tank. Place the optical fiber into the water tank and then heat the water. The change of temperature and Brillouin frequency shift during the whole heating process are recorded, and the linear relationship between temperature and Brillouin frequency shift is learned. The temperature factor is $1.775 \mathrm{MHz} /{ }^{\circ} \mathrm{C}$ and the laboratory calibration relationship between temperature and Brillouin frequency shift is shown in Figure 5.

\section{Field Application and Results}

3.1. Description of the Study Site. The monitoring site is located at overlying coalbed methane (CBM) drainage roadway of 1413A longwall panel of Zhangji coal mine (Huainan Mining Industry), China. The roadway has a length of 


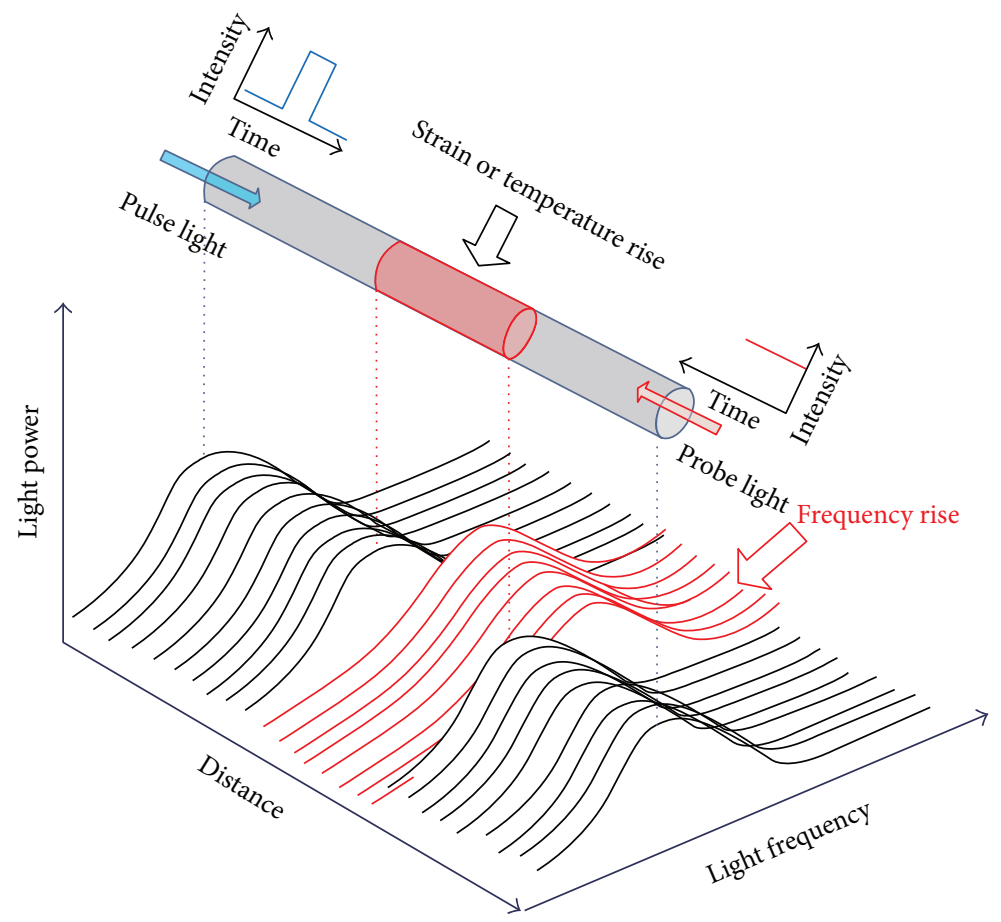

FIGURE 1: A schematic view of time and frequency domain acquisition for strain or temperature detection using BOTDR [28].

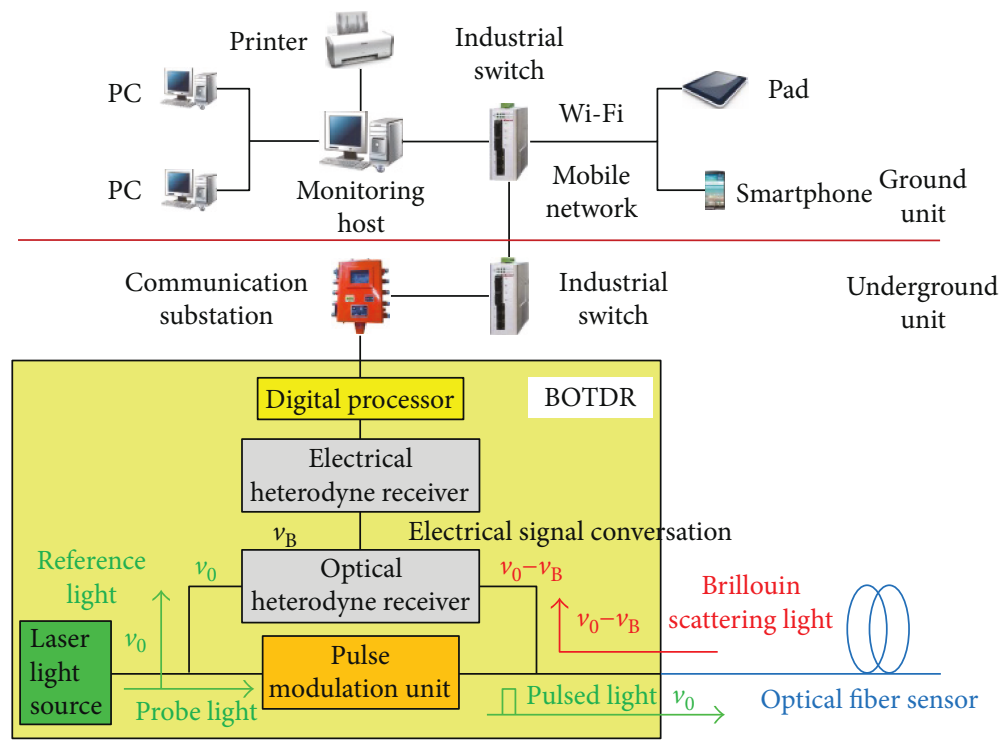

FIgURE 2: The BOTDR-based surrounding rock deformation monitoring system installed in an underground coal mine.

$1598 \mathrm{~m}$, a diameter of $4.5 \mathrm{~m}$, and a buried depth of $505 \mathrm{~m}$. the roadway was excavated by a gripper TBM.

The CBM drainage roadway is situated in coal-bearing strata which consist of fine sandstone, medium sandstone, argillaceous sandstone, and number 1 coal seam. The geometry of CBM drainage roadway cross-section is a circle with a diameter of $4.5 \mathrm{~m}$ and the roadway is supported by rockbolts and steel meshes. The number 1 coal seam is $6.5 \mathrm{~m}$ in thickness with a dip angle of $2-3^{\circ}$ and situated $25-30 \mathrm{~m}$ beneath the CBM drainage roadway. The location of Zhangji coal mine and geological setting of the CBM drainage roadway are illustrated in Figure 6. The roadway surrounding rock mainly consists of sandstone, and the rock properties have been obtained through laboratory tests. The rock properties are shown in Table 1.

The ground stress data had been obtained by borehole stress relief measurements. The measurement results suggested that the ground stress is controlled by tectonic stress. The orientation of the maximum horizontal stress is $116.3^{\circ}$ (NWW-SEE), and the magnitude of vertical stress, the minimum horizontal stress, and the maximum horizontal stress are 14.5 MPa, 13.4 MPa, and 37.4 MPa, respectively, 


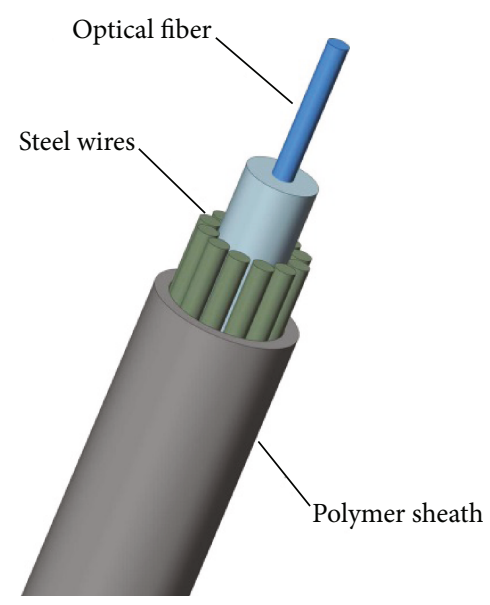

Figure 3: Schematic view of steel wire reinforced optical fiber sensor.

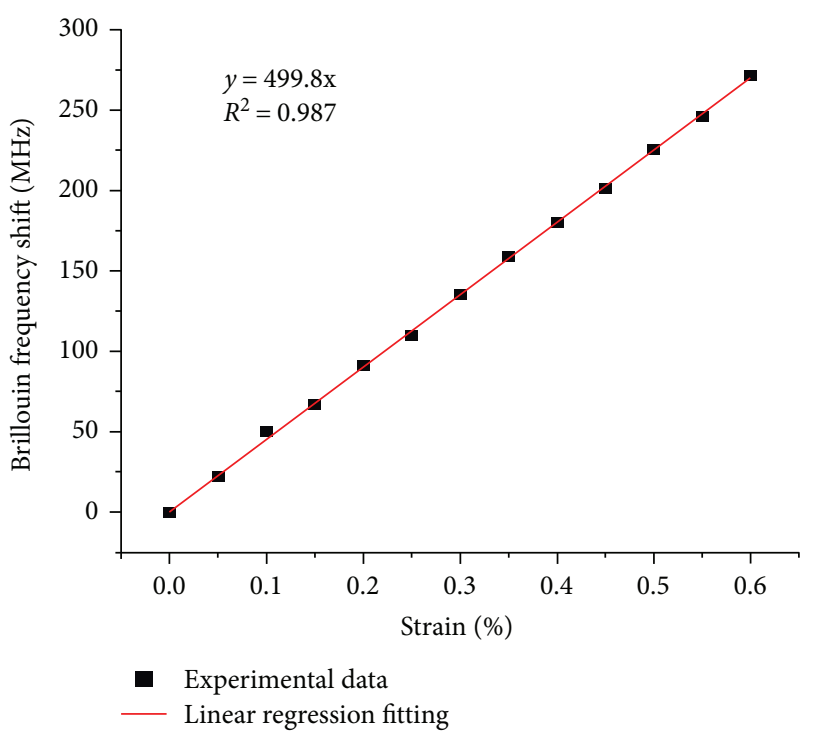

Figure 4: Brillouin frequency shift versus strain in laboratory calibrations.

$\sigma_{\mathrm{v}}: \sigma_{\mathrm{h}}: \sigma_{\mathrm{H}}$ ratio approximately at $1.08: 1: 2.8$. This direction of maximum horizontal stress is in agreement with local geological setting.

It is the first application of TBM in roadway excavation project in an underground coal mine which is operated by vertical shafts. The surrounding rock deformation behaviors of TBM-excavated roadway are estimated to be different with that of roadways constructed by conventional drilling and blasting due to different excavation disturbance effects of two excavation techniques. The monitoring works were conducted for studying deformation and stress filed redistribution behaviors of surrounding rocks of the TBM-excavated roadways, and the monitoring results can provide a reference for surrounding rock control, safety evaluation, and roadway support design.

3.2. Sensor Layout. The excavation works typically result in deformations and damages of roadway surrounding rocks,

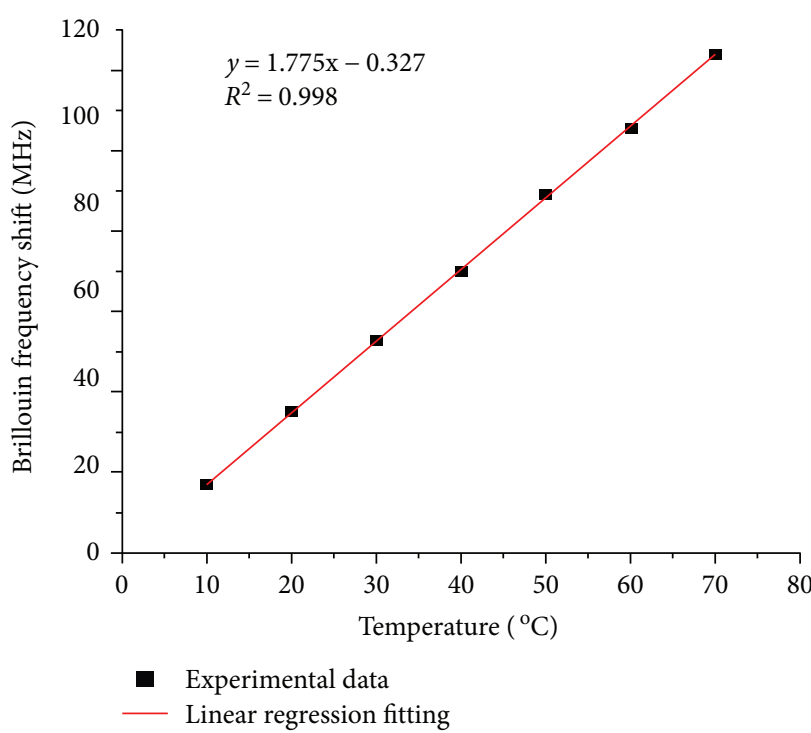

FIGURE 5: Brillouin frequency shift versus temperature in laboratory calibrations.

and continuous deformations and damages occur on both surface and interior section of surrounding rocks under the effect of the disturbed stress field. During the deformation and damage processes, the fractures within the interior sections of the surrounding rocks could expand themselves to the surface of surrounding rocks and result in damages or collapses of roadways. Therefore, the deformation and damage behaviors of roadway surrounding rocks are demanded to evaluate the stability of roadways during the excavation and utilization of roadways. In previous researches, optical fiber sensors were typically installed behind excavation faces within excavated roadways and the strain data of roadway surface can be obtained after excavation works. Nevertheless, the conventional monitoring systems cannot record strain variation during excavation works and the strain distribution in the interior sections of surrounding rocks.

In order to understand disturbance behaviors of roadway surrounding rocks under TBM excavation, two monitoring boreholes were drilled from an adjacent roadway (main gate of 1413A longwall panel) to the CBM drainage roadway of 1413A longwall panel. Optical fiber sensors were installed within the boreholes; therefore, as the TBM pass through the monitoring area, the strain values at various depths of roadway surrounding rocks along the radial direction of the roadway can be detected. As shown in Figure 7, two monitoring boreholes were set between the TBM-excavated roadway and its adjacent roadway with a space of $45 \mathrm{~m}$. The horizontal and vertical distance of the two sides of monitoring boreholes are $40 \mathrm{~m}$ and $26.5 \mathrm{~m}$, respectively. Both two boreholes have a length of $48 \mathrm{~m}$ and dip angle of boreholes is $34^{\circ}$. In future monitoring operations, the positions, number, and space of sensors can be determined based on the demands of monitoring and site conditions.

3.3. Sensor Installation. Optical fiber sensors were installed with monitoring boreholes. The diameter of boreholes should fulfill the requirements of sensor installation. In this 


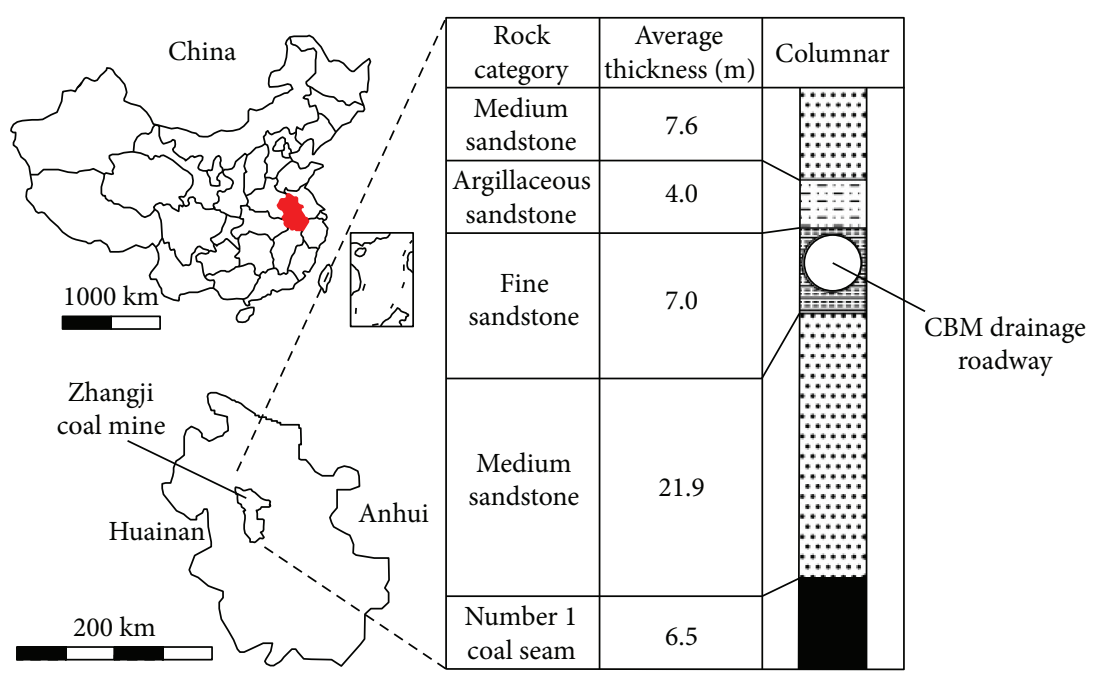

FIGURE 6: Location and strata histogram of CBM drainage roadway in Zhangji coal mine.

TABLE 1: Rock properties of roadway surrounding rocks.

\begin{tabular}{lcccccc}
\hline $\begin{array}{l}\text { Rock } \\
\text { category }\end{array}$ & $\begin{array}{c}\text { Density } \\
\left(\mathrm{g} / \mathrm{cm}^{3}\right)\end{array}$ & $\begin{array}{c}\mathrm{UCS}^{1} \\
(\mathrm{MPa})\end{array}$ & $\begin{array}{c}\text { Tensile strength } \\
(\mathrm{MPa})\end{array}$ & $\begin{array}{c}\text { Elastic modulus } \\
(\mathrm{GPa})\end{array}$ & $\begin{array}{c}\text { Limited tensile strain } \\
(\mu \varepsilon)\end{array}$ & $\begin{array}{c}\text { Limited compress strain } \\
(\mu \varepsilon)\end{array}$ \\
\hline Sandstone & 2.51 & 28 & 7.8 & 42 & 185.7 & 666.7 \\
\hline
\end{tabular}

${ }^{1}$ Uniaxial compressive strength.

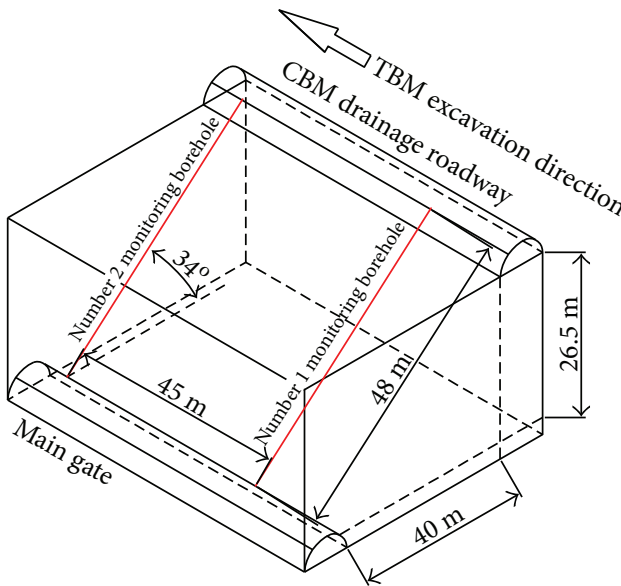

FIGURE 7: The monitoring borehole layout.

study, two boreholes with a diameter of $127 \mathrm{~mm}$ were drilled from the main gate of 1413A longwall panel for optical fiber sensor installation. The distance from the main gate entrance to the number 1 borehole and number 2 borehole is $867 \mathrm{~m}$ and $912 \mathrm{~m}$, respectively. follows:

The installation procedure of optical fiber sensors is as

(1) Installing the optical fiber sensors: Tie the steel wirereinforced optical fiber sensor on a polyvinyl chloride (PVC) tube which has a diameter of $40 \mathrm{~mm}$ then place the PVC tube into the borehole. The PVC tube is used as an orienting device for optical fiber sensors.
(2) Place the exhaust pipe and grouting pipe into the borehole and seal the borehole by installing a sealing plate.

(3) Start grouting. Injecting the cement grout into the borehole, after the cement hardens, it is capable of providing protection for optical fiber sensors as well as insurance of essential coupling effect between sensors and surrounding rocks.

(4) Connect transmission lines and power supplies. The preparation of monitoring works is finished. The structure of monitoring boreholes is shown in Figure 8 .

3.4. Monitoring Results and Analysis. Monitoring works were started after cement grout curing. The initial strain along the optical fiber sensors was recorded as reference values. Therefore, quantitative evaluation of damage degree of surrounding rocks under the disturbed effects of TBM excavation can be studied by measuring the changing of strain values. The limited compressive and tensile strain of rocks had been obtained from laboratory tests of rock specimens. Once the strain values exceed the limited strains, it can be deemed that damages occur in surrounding rocks and the positions of damages can be detected. Consequently, an early warning of surrounding rock damages can be provided.

The optical fiber sensors were set on January 28, 2015, and the monitoring works finished on March 1, 2015. The strain values of surrounding rocks which were induced by TBM excavation has been clearly measured by optical fiber 


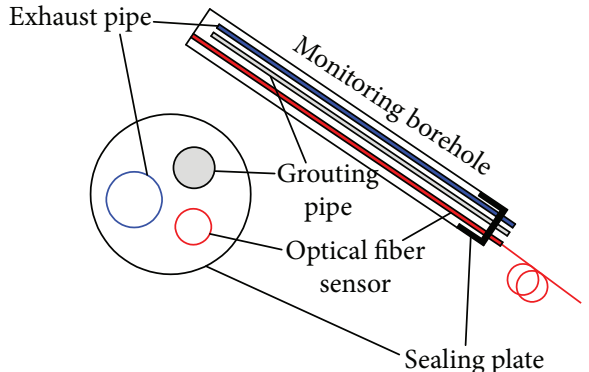

Figure 8: A schematic view of monitoring borehole and optical fiber sensor installation.

sensors when the TBM passed through the monitoring area. The monitoring results of numbers 1 and 2 boreholes are shown in Figures 9 and 10, respectively.

The TBM approached the number 1 borehole firstly. In the 8th of February, the TBM is $12 \mathrm{~m}$ to the number 1 borehole, the tensile strain increased to $629.85 \mu \varepsilon$ at a depth of $35.8 \mathrm{~m}$ in the number 1 borehole, and the compress strain ascended to $-649.2 \mu \varepsilon$ in $43.3 \mathrm{~m}$. From February 9 to February 11, the TBM passed through the monitoring area of the number 1 borehole. The maximum tensile strain happened at a depth of $35.9 \mathrm{~m}$ with the tensile strain value of $879.4 \mu \varepsilon$, and the maximum compressive strain occurred at a depth of $43.2 \mathrm{~m}$ with the compressive strain magnitude of $-865.1 \mu \varepsilon$. After February 12, as the TBM passed through the borehole and advanced forward, the strain fell back to normal values which range from $100 \mu \varepsilon$ to $-100 \mu \varepsilon$.

Figure 10 indicates that strain data obtained from the number 2 borehole are in agreement with monitoring results of the number 1 borehole. The TBM passed through the number 2 borehole in February 12 and that resulted in the increasing of surrounding rock strains from February 12 to February 15. The maximum tensile strain was detected at a depth of $35.4 \mathrm{~m}$ in the number 1 borehole with a value of $660.7 \mu \varepsilon$ and the maximum compressive strain was measured at a depth of $42.2 \mathrm{~m}$ with a value of $-623.6 \mu \varepsilon$. After February 16, the roadway surrounding rock strain fell back to a low level.

According to the monitoring results of two optical fiber sensors, the maximum tensile and compressive strain were detected at depth of $35.9 \mathrm{~m}$ and $43.2 \mathrm{~m}$ in the number 1 borehole and $35.4 \mathrm{~m}$ and $42.2 \mathrm{~m}$ in the number 2 borehole, respectively. In this study, sites at a depth of $35.5 \mathrm{~m}$ and $43 \mathrm{~m}$ within both monitoring boreholes were chosen as critical monitoring points of tensile and compress concentration. Figure 11 illustrates the relationship between TBM advancing and strain changing at tensile and compressive concentration points within two boreholes. In the figure, $\Delta x=0$ represents the arrival of TBM face at the monitoring plane, while the distance of the TBM behind and ahead the monitoring plane is indicated as $-\Delta \mathrm{x}$ and $+\Delta \mathrm{x}$, respectively. As shown in Figure 11, when $\Delta \mathrm{x}=5 \mathrm{~m}$, both tensile and compressive strain increased significantly. As TBM passed through the borehole, the strain values remained on a high level for 3 to 4 days, and then the strain values dropped back to normal level $(-100 \mu \varepsilon$ to $100 \mu \varepsilon)$ when $\Delta \mathrm{x}=-40 \mathrm{~m}$.

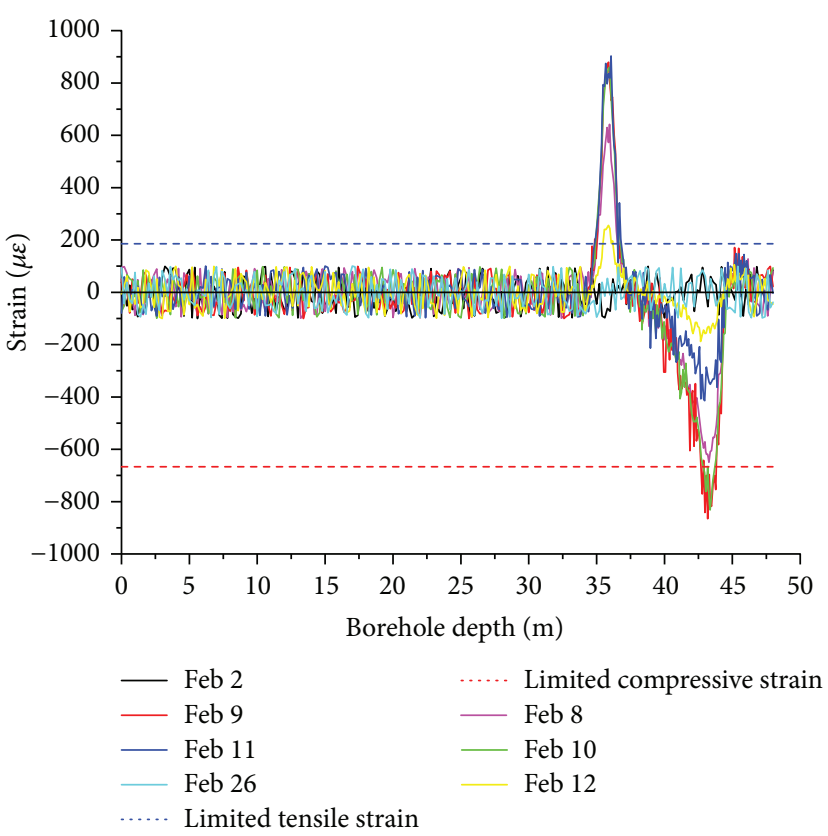

FIGURE 9: Monitoring results of surrounding rock strain along the optical fiber in the number 1 borehole during the approaching of the TBM.

It can be learned from the monitoring results that the disturbance range ahead the TBM excavation face is around $5 \mathrm{~m}$. There are tensile concentration zone and compressive concentration zone located in $35.5 \mathrm{~m}$ and $43 \mathrm{~m}$ depth within the boreholes. Comparing the monitoring results with the limited tensile and compressive strain which obtained from laboratory tests, the TBM-induced damage zone can be acquired in real-time by taking advantage of the BOTDRbased online monitoring system. Damage zones had been detected about $5 \mathrm{~m}$ and $12.5 \mathrm{~m}$ away from roadway surface, and these damage zones cannot be found by conventional techniques such as extensometers or instrumental bolts due to their limited monitoring ranges. The strain values within $5 \mathrm{~m}$ to roadway surface are relatively low due to the effect of roadway support facilities such as rockbolt and cable bolt. The monitoring results also indicate that the roadway surrounding rock disturbance mainly happened after excavation. The surrounding rock typically gets rebalance within 3-4 days after excavation and that indicates the excavation disturbance of TBM is significantly less than that of conventional drilling and blasting.

\section{Discussions}

Compared with conventional monitoring techniques, optical fibers which are used in BOTDR-based monitoring system are both sensors and propagation medium of signals. One optical fiber can detect strain in a large area of surrounding rocks and the monitoring area can be controlled by simply adding or reducing the number of optical fibers. The disturbance and damage behaviors of rocks under TBM excavation had been learned by using BOTDR-based monitoring system. The BOTDR monitoring system is capable of 


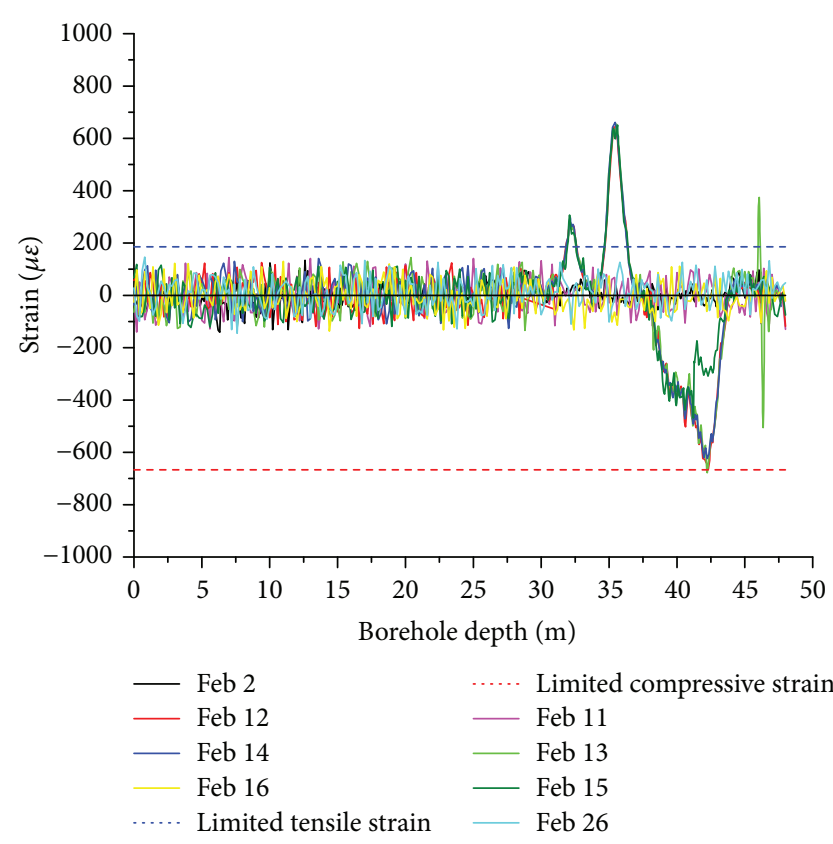

FIGURE 10: Monitoring results of surrounding rock strain along the optical fiber in the number 2 borehole during the approaching of the TBM.

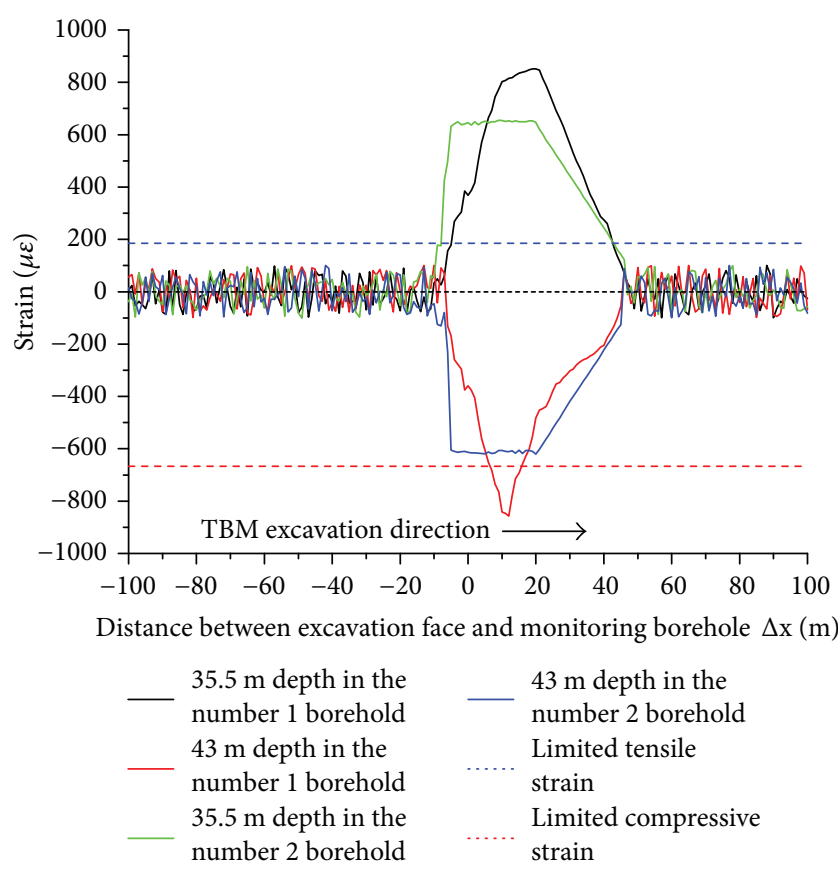

FIGURE 11: Strain variation of surrounding rocks along with TBM advancing.

measuring $\pm 15000 \mu \varepsilon$ with an accuracy of $\pm 15 \mu \varepsilon$. In contrast, the resolution of the mechanical monitoring devices such as extensometers typically is $1 \mathrm{~mm}$ in a measuring range of $5 \mathrm{~m}$, which is equivalent to $200 \mu \varepsilon$ (strain). The accuracy of the BOTDR-based monitoring system is significantly higher than that of conventional monitoring devices.
For discrete strain monitoring techniques (FBG, electric resistance strain gauges, vibrating wire strain gauge, etc.), it is difficult to obtain strain data over long distances because this requires installing a sufficient number of sensors and a corresponding increase in costs. The total cost of BOTDR monitoring operation in this study is $300,000 \mathrm{RMB}$, whereas if FBG sensors had been used as alternatives, in this case, the cost would increase to over $600,000 \mathrm{RMB}$ due to the high price of FBG sensors (700 RMB). Moreover, the cost of BOTDR monitoring could be even lower in future coal mine applications because abandoned boreholes (such as sampling boreholes, surveying boreholes, methane drainage boreholes, and water drainage boreholes) near roadways can be used to install optical fiber sensors.

In previous BOTDR monitoring applications in coal mines, optical fiber sensors were typically installed on the rock surface. Therefore, the deformation and damage behaviors of surrounding rocks are hardly acquired by the conventional layout of sensors. Installing sensors within boreholes which are capable of providing well protection of sensors and the installation works can be conducted in adjacent roadways so that the interference between sensor installation and roadway supporting works can be avoided.

\section{Conclusions}

A BOTDR-based monitoring system has been proposed and successfully applied in Zhangji coal mine, China. An accurate, reliable, large-scale, and real-time monitoring of surrounding rocks had been obtained by means of the special layout of sensors and installing optical fiber sensors within boreholes. The deformation and damage behaviors of surrounding rocks under the disturbance of TBM excavation had been learned. An early warning of surrounding rock damages can be provided when the strain values of rocks exceed the limits and additional roadway supporting works can be conducted. Consequently, the preventionoriented strategy of roadway safety and stability control can be realized.

\section{Conflicts of Interest}

The authors declare no conflict of interest.

\section{Acknowledgments}

The work presented in this paper is financially supported by the National Natural Science Foundation of China (Grant nos. 51674006 and 51474004) and Youth Fund of Anhui University of Science and Technology (Grant nos. QN2017211 and QN2017222). The authors would like to express their appreciation to the staff of Zhangji coal mine for their substantial support.

\section{References}

[1] H. Zhou, C. Qu, D. Hu et al., "In situ monitoring of tunnel deformation evolutions from auxiliary tunnel in deep mine," Engineering Geology, vol. 221, pp. 10-15, 2017. 
[2] S. Yang, M. Chen, H. Jing, K. Chen, and B. Meng, "A case study on large deformation failure mechanism of deep soft rock roadway in Xin'an coal mine, china," Engineering Geology, vol. 217, pp. 89-101, 2017.

[3] Q. Yin, H. Jing, D. Dai, T. Zhu, H. Zhao, and B. Meng, "Cabletruss supporting system for gob-side entry driving in deep mine and its application," International Journal of Mining Science and Technology, vol. 26, no. 5, pp. 885-893, 2016.

[4] B. Nie, X. Huang, X. Sun, and A. Li, "Experimental study on physiological changes of people trapped in coal mine accidents," Safety Science, vol. 88, pp. 33-43, 2016.

[5] Y.Zhao, T. Yang, P. Zhang, J. Zhou, Q. Yu, and W. Deng, “The analysis of rock damage process based on the microseismic monitoring and numerical simulations," Tunnelling and Underground Space Technology, vol. 69, pp. 1-17, 2017.

[6] Z. Zhao, Y. Zhang, C. Li et al., "Monitoring of coal mine roadway roof separation based on fiber Bragg grating displacement sensors," International Journal of Rock Mechanics and Mining Sciences, vol. 74, pp. 128-132, 2015.

[7] V. Kajzar, R. Kukutsch, P. Waclawik, and J. Nemcik, "Innovative approach to monitoring coal pillar deformation and roof movement using 3d laser technology," Procedia Engineering, vol. 191, pp. 873-879, 2017.

[8] W. Yu, W. Wang, X. Chen, and S. Du, "Field investigations of high stress soft surrounding rocks and deformation control," Journal of Rock Mechanics and Geotechnical Engineering, vol. 7, no. 4, pp. 421-433, 2015.

[9] J. B. Martino and N. A. Chandler, "Excavation-induced damage studies at the underground research laboratory," International Journal of Rock Mechanics and Mining Sciences, vol. 41, no. 8, pp. 1413-1426, 2004.

[10] P. Blümling, F. Bernier, P. Lebon, and C. Derek Martin, "The excavation damaged zone in clay formations time-dependent behaviour and influence on performance assessment," Physics and Chemistry of the Earth, Parts $A / B / C$, vol. 32, no. 8-14, pp. 588-599, 2007.

[11] Z. Lubosik, P. Waclawik, P. Horak, and A. Wrana, "The influence of in-situ rock mass stress conditions on deformation and load of gateroad supports in hard coal mine," Procedia Engineering, vol. 191, pp. 975-983, 2017.

[12] L. Sheng-dong, Z. Ping-song, C. Yu, and W. Bo, "Characteristic of geological anomaly detected by combined geophysical methods in a deep laneway of coal mine," Procedia Earth and Planetary Science, vol. 1, no. 1, pp. 936-942, 2009.

[13] E. D. Guy, R. C. Nolen-Hoeksema, J. J. Daniels, and T. Lefchik, "High-resolution sh-wave seismic reflection investigations near a coal mine-related roadway collapse feature," Journal of Applied Geophysics, vol. 54, no. 1-2, pp. 51-70, 2003.

[14] A. Motil, A. Bergman, and M. Tur, "[INVITED] State of the art of Brillouin fiber-optic distributed sensing," Optics \& Laser Technology, vol. 78, pp. 81-103, 2016.

[15] S. A. P. de Jong, J. D. Slingerland, and N. C. van de Giesen, "Fiber optic distributed temperature sensing for the determination of air temperature," Atmospheric Measurement Techniques, vol. 8, no. 1, pp. 335-339, 2015.

[16] T. R. C. Aston, J. L. Gilby, and C. M. K. Yuen, “A comparison of rock mass disturbance in TBM and drill and blast drivages at the Donkin mine, Nova Scotia," International Journal of Mining and Geological Engineering, vol. 6, no. 2, pp. 147$162,1988$.
[17] L. Tartara, C. Codemard, J.-N. Maran, R. Cherif, and M. Zghal, "Full modal analysis of the Brillouin gain spectrum of an optical fiber," Optics Communication, vol. 282, no. 12, pp. 2431-2436, 2009.

[18] H. Naruse, H. Uehara, T. Deguchi et al., "Application of a distributed fibre optic strain sensing system to monitoring changes in the state of an underground mine," Measurement Science and Technology, vol. 18, no. 10, pp. 3202-3210, 2007.

[19] G. Cheng, B. Shi, H. H. Zhu, C. C. Zhang, and J. H. Wu, "A field study on distributed fiber optic deformation monitoring of overlying strata during coal mining," Journal of Civil Structural Health Monitoring, vol. 5, no. 5, pp. 553-562, 2015.

[20] D. Zhang, B. Shi, and H. Z. Xu“, "The BOTDR-based strain monitoring for tunnel," Journal of Engineering Geology, vol. 12, no. 4, pp. 422-426, 2004.

[21] S. Wang and L. Luan, "Analysis on the security monitoring and detection of mine roof collapse based on BOTDR technology," in International Conference on Soft Computing in Information Communication Technology, pp. 83-86, Taipei, China, 2014.

[22] E. Hoek, "Big tunnels in bad rock," Journal of Geotechnical and Geoenvironmental Engineering, vol. 127, no. 9, pp. 726-740, 2001.

[23] V. A. Kontogianni and S. C. Stiros, "Predictions and observations of convergence in shallow tunnels: case histories in Greece," Engineering Geology, vol. 63, no. 3-4, pp. 333-345, 2002.

[24] M. J. Kavvadas, "Monitoring ground deformation in tunnelling: current practice in transportation tunnels," Engineering Geology, vol. 79, no. 1-2, pp. 93-113, 2005.

[25] H. Naruse, K. Komatsu, K. Fujihashi, and M. Okutsu, "Telecommunications tunnel monitoring system based on distributed optical fiber strain measurement," in 17th International Conference on Optical Fibre Sensors, Bruges, Belgium, 2005.

[26] C.-Y. Hong, Y.-F. Zhang, G.-W. Li, M.-X. Zhang, and Z.-X. Liu, "Recent progress of using Brillouin distributed fiber optic sensors for geotechnical health monitoring," Sensors and Actuators A: Physical, vol. 258, pp. 131-145, 2017.

[27] J. S. Zhang, Y. Q. Li, and S. Zhang, "Performance analysis of temperature and strain simultaneous measurement system based on heterodyne detection of Brillouin scattering," in 2008 1st Asia-Pacific Optical Fiber Sensors Conference, vol. 6, pp. 1-5, Chengdu, China, 2008.

[28] H. Ohno, H. Naruse, M. Kihara, and A. Shimada, "Industrial applications of the BOTDR optical fiber strain sensor," Optical Fiber Technology, vol. 7, no. 1, pp. 45-64, 2001.

[29] H. H. Kee, G. P. Lees, and T. P. Newson, "All-fiber system for simultaneous interrogation of distributed strain and temperature sensing by spontaneous Brillouin scattering," Optics Letters, vol. 25, no. 10, pp. 695-697, 2000.

[30] K. Hotate and M. Tanaka, "Distributed fiber Brillouin strain sensing with $1-\mathrm{cm}$ spatial resolution by correlation-based continuous-wave technique," IEEE Photonics Technology Letters, vol. 14, no. 2, pp. 179-181, 2002.

[31] C. Y. Hong, J. H. Yin, H. F. Pei, and D. S. Xu, "In application of fiber optic sensors in pullout testing of model soil nails," in The Asian Regional Conference on Soil Mechanics and Geotechnical Engineering, p. 444, Hong Kong, 2011.

[32] P. Wright, "Assessment of London underground tube tunnels - investigation, monitoring and analysis," Smart Structures and Systems, vol. 6, no. 3, pp. 239-262, 2010. 
[33] G. Wei, "BOTDR based distributed strain test on bored pile buried in complicated geological ground," Journal of Engineering Geology, vol. 16, pp. 826-832, 2008.

[34] Z. Zhou, J. He, M. Huang, J. He, and G. Chen, "Casing pipe damage detection with optical fiber sensors: a case study in oil well constructions," Advances in Civil Engineering, vol. 2010, Article ID 638967, 9 pages, 2010. 


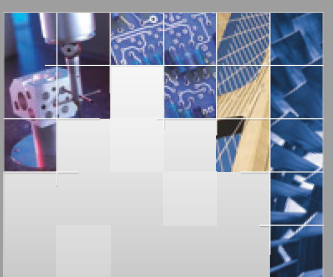

\section{Enfincering}
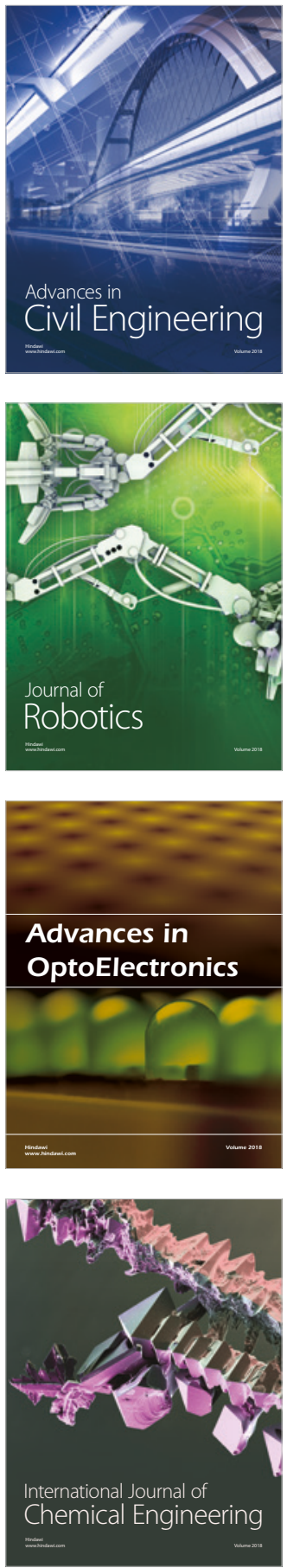

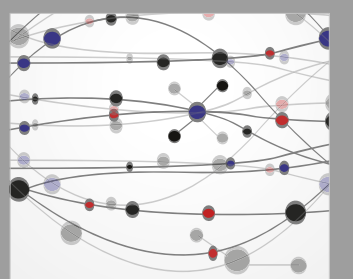

\section{Rotating \\ Machinery}

The Scientific World Journal

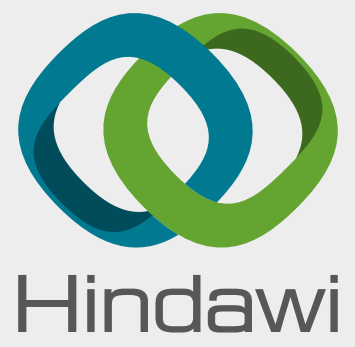

Submit your manuscripts at

www.hindawi.com
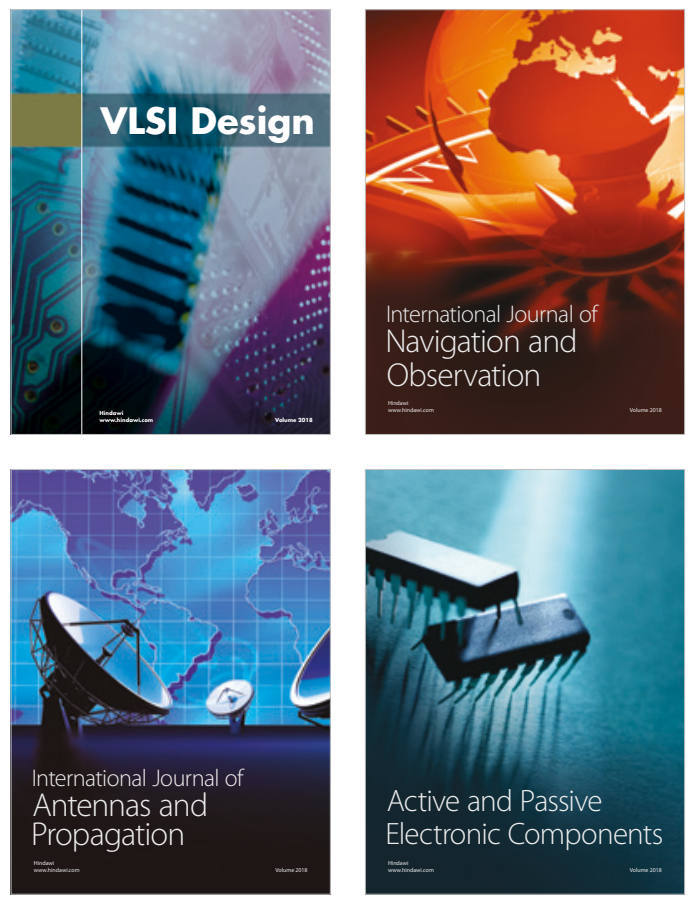
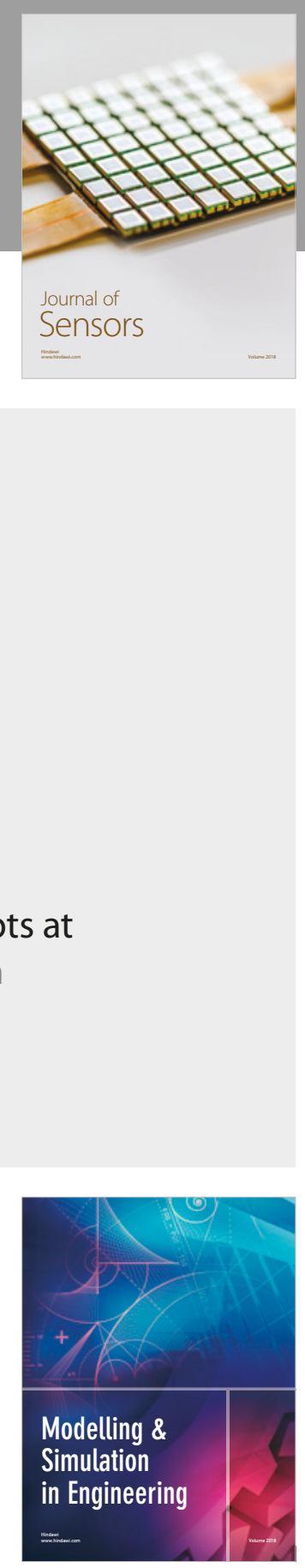

\section{Advances \\ Multimedia}
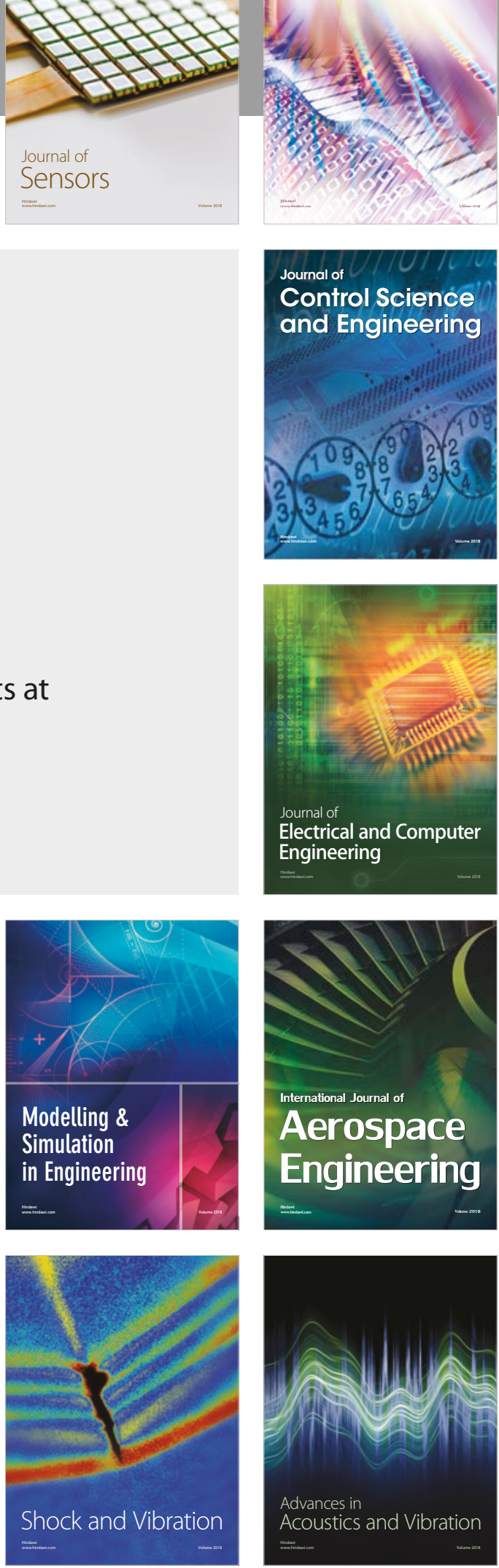